\title{
Interface for Dynamic Modification of the Transformation Parameters of the PSOLA Algorithm"
}

\author{
Lyes Demri*, Leila Falek, Hocine Teffahi ${ }^{1}$
}

\begin{abstract}
The prosody of a speech signal is related to many factors: the social and geographical origin of the speaker, his or her emotional state, his physiological state (weariness, sickness, ...) and the type of the sentence (interrogative, affirmative, etc.). A good synthesis or speech transformation system must account for all of these factors in order to produce a speech that sounds natural. In this paper, we propose a graphical interface for the modification of the prosodic features of the speech signal (the melodic curve fundamental frequency and temporal

organization of the syllables - and the formantic trajectories) using the PSOLA algorithm. The interface allows the user to manually introduce the desired trajectories of the transformation parameters of the PSOLA algorithm in order to produce a transformed signal which has the desired prosody. The results are acceptable, especially for the modification of the fundamental frequency and of the temporal organization of the source signal.
\end{abstract}

\section{Keywords: Speech Synthesis, PSOLA Algorithm, PSOLA Parameters, Prosodic Parameters, Dynamically Changing, Interface}

\section{Introduction}

The ability to modify the acoustic features of a voice is an important factor in voice transformation and synthesis systems. It is commonly accepted that a large part of the information contained in a speech signal lies in the prosody (the melodic curve, that is, the evolution of the fundamental frequency F0 and the temporal organization of the syllables), and in the voice quality (represented by the formants of the spectral envelope) ([1], [2]). These features inform us both about the type of sentence (affirmative, interrogative, exclamative), and the emotional state of the speaker (sadness, happiness, anger, etc.).

In this study, we focus on commanding those features through an interactive interface between the user and the transformation system which is built on the PSOLA algorithm. The PSOLA algorithm is based on the calculation of three transformation parameters, $\alpha, \beta$ and $\gamma$, which correspond respectively to the temporal organization, the fundamental frequency, and the voice quality.

\section{The PSOLA Algorithm}

The PSOLA algorithm (Pitch Synchronous Overlap-Add) is well known in the domains of voice transformation and synthesis. It is used to modify the intrinsic characteristics of a voice signal [4], [8]. The PSOLA algorithm is based on an analysis/synthesis scheme and can modify the signal both in the time and the frequency domains [3]. Here is a brief description of the algorithm.

\subsection{Analysis}

The analysis step consists of placing reading marks mi on the original signal $s(t)$, according to the local characteristics of its components (periodic, random, transitory). These marks are placed

\footnotetext{
${ }^{1}$ Electronics and Computer Science Department, USTHB, BP 32, BabEzzouar, Algiers, Algeria.

* Corresponding Author: Email: ldemri1987@hotmail.fr

\# This paper has been presented at the International Conference on

Advanced Technology\&Sciences (ICAT'14) held in Antalya (Turkey), August 12-15, 2014.
}

on the signal's local maxima of energy, and usually correspond to the impulsions in the source-filter scheme, and to the glottal impulsions in the case of a voice [9]. The signal is thus segmented into elementary signals through the use of Hanning windows hi(t) whose lengths are usually 2 or 4 times that of the fundamental frequency and centered on the mi [10]. Each of the windows must be centered on the the local maximum of energy. The resulting signal si(t) can be written as :

$$
s_{i}(t)=h_{i}\left(t-m_{i}\right) s(t)
$$

The analysis algorithm is as follows (figure 1):

- Determination of the pitch period $\mathrm{P}(\mathrm{t})$ of the input signal and of time instants (pitch marks) ti. These pitch marks are in correspondence with the maximum amplitude or glottal pulses at a pitch synchronous rate during the periodic part of the sound and at a constant rate during the unvoiced portions. In practice $\mathrm{P}(\mathrm{t})$ is considered constant $\mathrm{P}(\mathrm{t})=\mathrm{P}(\mathrm{ti})=\mathrm{t}+\mathrm{t}-\mathrm{ti}$ on the time interval (ti, ti+l).

- Extraction of a segment centered at every pitch mark ti by using a Hanning window with the length $\mathrm{Li}=2 \mathrm{P}$ (ti) (two pitch periods) to ensure fade-in and fade-out.

\subsection{Synthesis}

The synthesis step consists in placing writing marks which maintain the same distances between each other as those of the reading marks, so that the original fundamental frequency is preserved. The correspondence index, which is the corresponding position to the writing marks in the original signal, allows us to select the elementary signal which will be used at this writing mark (an already existing elementary signal (TD-PSOLA), or interpolated in the time domain (TDI-PSOLA), or in the frequency domain (FDI-PSOLA) [10]).

Synthesis algorithm (figure 2): for every synthesis pitch mark $\widetilde{\mathrm{k}}$ 1. Choice of the corresponding analysis segment $i$ (identified by the time mark ti) minimizing the time distance $|\alpha \mathrm{ti}-\widetilde{\mathrm{tk}}|$.

2. Overlap and add the selected segment. Notice that some input segments will be repeated for $\alpha>1$ (time expansion) or discarded when $\alpha<1$ (time compression). 
3. Determination of the time instant $\sqrt{\mathrm{k}+1}$ where the next synthesis segment will be centered, in order to preserve the local pitch, by the relation

$$
\hat{t}_{k+1}=\tilde{t}_{k}+\tilde{P}\left(\tilde{t}_{k}\right)=\tilde{t}_{k}+P\left(t_{i}\right)
$$

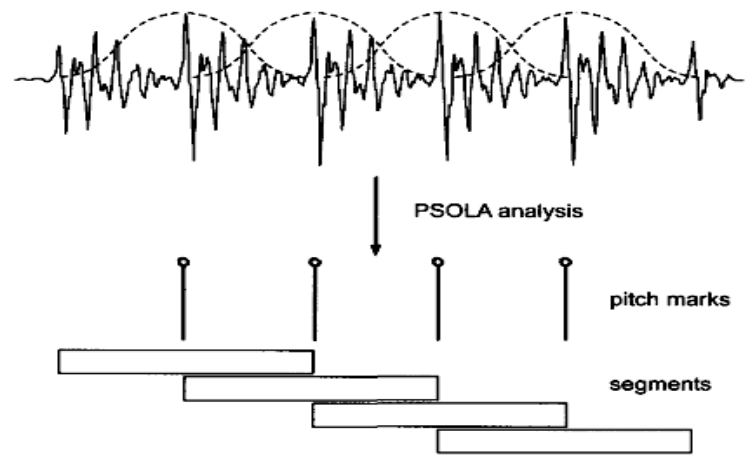

Figure 1. PSOLA pitch analysis

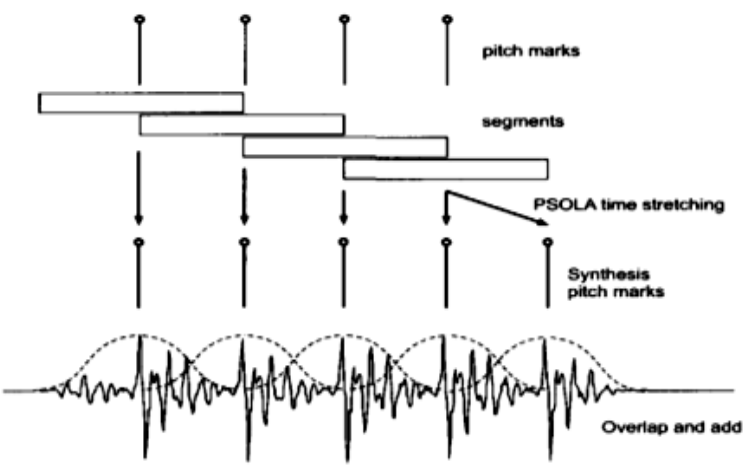

Figure 2. PSOLA synthesis for time stretching

It should be noticed that the determination of the pitch and of the position of pitch marks is not a trivial problem and could be difficult to implement robustly in real-time. Stretching factors typically range from $\alpha=0.25$ to 2 for speech. Audible buzziness appears in unvoiced sound when larger values are applied, due to the regular repetition of identical input segments. In order to prevent the algorithm from introducing such an artificial shortterm correlation in the synthesis signal, it is advisable to reverse the time axis of every repeated version of an unvoiced segment. With such an artifice, speech can be slowed down by a factor of four, even though some tonal effect is encountered in voiced fricatives, which combine voiced and unvoiced frequency regions and thus cannot reversed in time.

A further effect that can be obtained by a variation of PSOLA is linear scaling of formant frequencies (see figure 3). In fact, we saw that a time scale of a signal corresponds to an inverse frequency scale. Thus when we perform time scaling of the impulse response of a filter, we inversely scale the frequency of formants. In PSOLA terms, this corresponds to time scaling the selected input segments before overlap and add in the synthesis step, without any change in the pitch marks calculation. To increase the frequencies of formants by a factor $\gamma$, every segment should be shortened by a factor $1 / \gamma$ by resampling. For example, the average formant frequencies of female adults are about 16 percent higher than those of male adults, and children's formants are about 20 percent higher than female formants. Notice that care should be taken when the frequencies increase in order to avoid foldover. Ideally bandlimited resampling should be used [12].
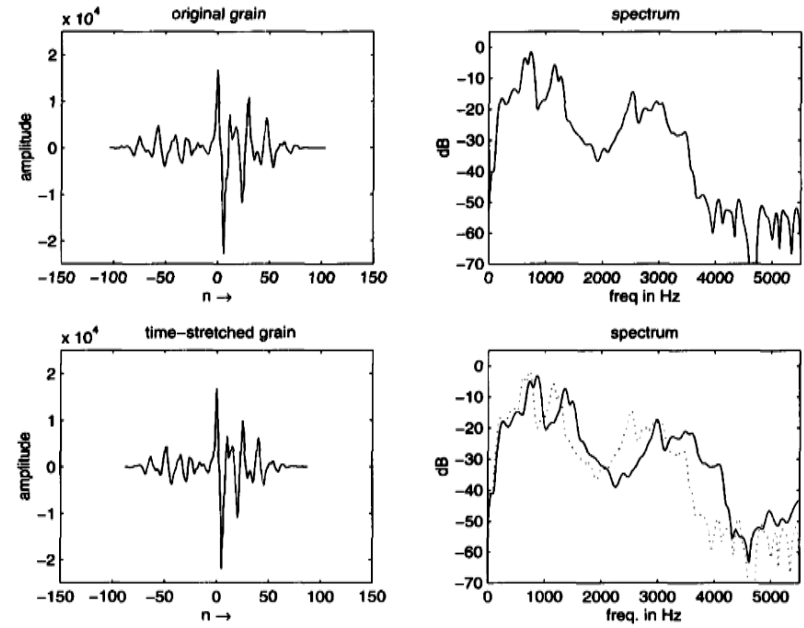

Figure 3. PSOLA: Variation of PSOLA as linear formant scaling

\section{Presentation of the Interface}

We have realized an interactive interface between the user and the computer that allows a dynamic modification of the transformation parameters of the PSOLA algorithm. The objective of the interface is to obtain a transformed signal from a source signal, through the modification of the three parameters cited above. The interface's inputs are:

the source signal

the trajectories for the three transformation parameters $\alpha, \beta$ and $\gamma$. The interface outputs the transformed signal. Here are the steps required to produce the transformed signal.

\subsection{Dynamic Modification of the Fundamental Frequency}

During the reconstruction step, the algorithm must calculate where the next waveform is to be placed. The position is given by the relation:

$$
t_{k}=t_{k}+(T / \beta)
$$

Where:

$t_{k}$ is the position of the local maximum of the current waveform, $\mathrm{T}$ is the period of the source signal at this instant,

$\beta$ is the shifting factor of the pitch.

For $\beta=1$, we therefore have:

$$
t_{k}=t_{k}+T
$$

In order to change the pitch in a dynamic way, we must introduce $\beta$ as varying during the reconstruction phase :

$$
t_{k}=t_{k}+(T / \beta(i))
$$

where $\beta$ is now a vector whose values are obtained by a simple linear interpolation between an initial point $\left(\beta \_\right.$ini) and a final point ( $\beta$ end). It is then necessary to send in only those two values to the PSOLA function which will automatically compute the values $\beta$ (i) of $\beta$ between these 2 points. With these values, the PSOLA algorithm can realize a dynamic modification of the pitch.

\subsection{Modification of $\alpha$}

It is not necessary for $\alpha$ to change dynamically like $\beta$. To illustrate this, let us take the example of a speech signal that contains 4 syllables « taratata » $(/ \mathrm{ta} /, / \mathrm{ra} /, / \mathrm{ta} /, / \mathrm{ta} /)$ that have the same lengths 
$(\mathrm{d} 1=\mathrm{d} 2=\mathrm{d} 3=\mathrm{d} 4)$. If we apply a dynamic $\alpha$ that varies linearly from 1 to 2 , the 4 syllables will have durations such that $\mathrm{d} 1<\mathrm{d} 2<$ $\mathrm{d} 3<\mathrm{d} 4$. The interface will behave just as if it segmented the original signal into 4 segments and applied a different value of $\alpha$ for each segment. It is therefore not necessary to introduce a vector of $\alpha$ (i) but simply to segment the input signal into many parts and apply a different $\alpha$ for each segment.

\subsection{Dynamic Modification of the Voice Quality $(\gamma)$}

The dynamic modification of the voice quality is similar to that of the pitch $(\beta)$. The user sends in a couple of values $\gamma_{-}$ini and $\gamma_{-}$end, and a linear interpolation between these two points generates the vector $\gamma$ that contains the values $\gamma(\mathrm{i})$ for each waveform.

\section{Experimental Results}

The interface has been realized in MATLAB and is illustrated in figure 4.

\subsection{Functioning Principle of the Graphical Interface}

The interface shows 4 graphs:

The speech signal

The desired variation curve for $\alpha$

The desired variation curve for $\beta$

The desired variation curve for $\gamma$

The desired trajectories for $\alpha, \beta$ and $\gamma$ can be realized either by manually introducing the values facing each graph, or by directly clicking on the graphs using the mouse where the points should be. Once the trajectories are introduced, the user can click "play» which will transform the source signal and playback the result. The interface first applies $\beta$, then $\gamma$, and then $\alpha$ (in this order). For each of the parameters, the signal is decomposed into segments. On each segment, a linear transformation is applied, as described in section 3. Once all the segments are transformed, they are concatenated to obtain the transformed signal. For each parameter the interface allows to save, load or erase the values of the parameters. Other click buttons allow the user to :

Select file: select a source signal in a directory.

Save transformed signal : save the transformed signal Play : Transform the source signal and play the result Play original signal : plays the source signal.

\subsection{Results Illustrations}

In this part, we present a few results obtained using the interface. The aim was to try and reproduce the prosody of a target sentence on a sentence that has equal length and same pitch syllables.

The target sentence is in french «oui pourquoi pas ?» uttered on the interrogative tone. The sentence is first recorded with the interrogative prosody - this will be the target sentence. The sentence is then recorded again on a neutral and monotonic tone (constant fundamental frequency and speech speed), as our source signal. The objective is to imitate the target by transforming the source file. The results obtained for the pitch and the temporal organization are given in figure 4 .

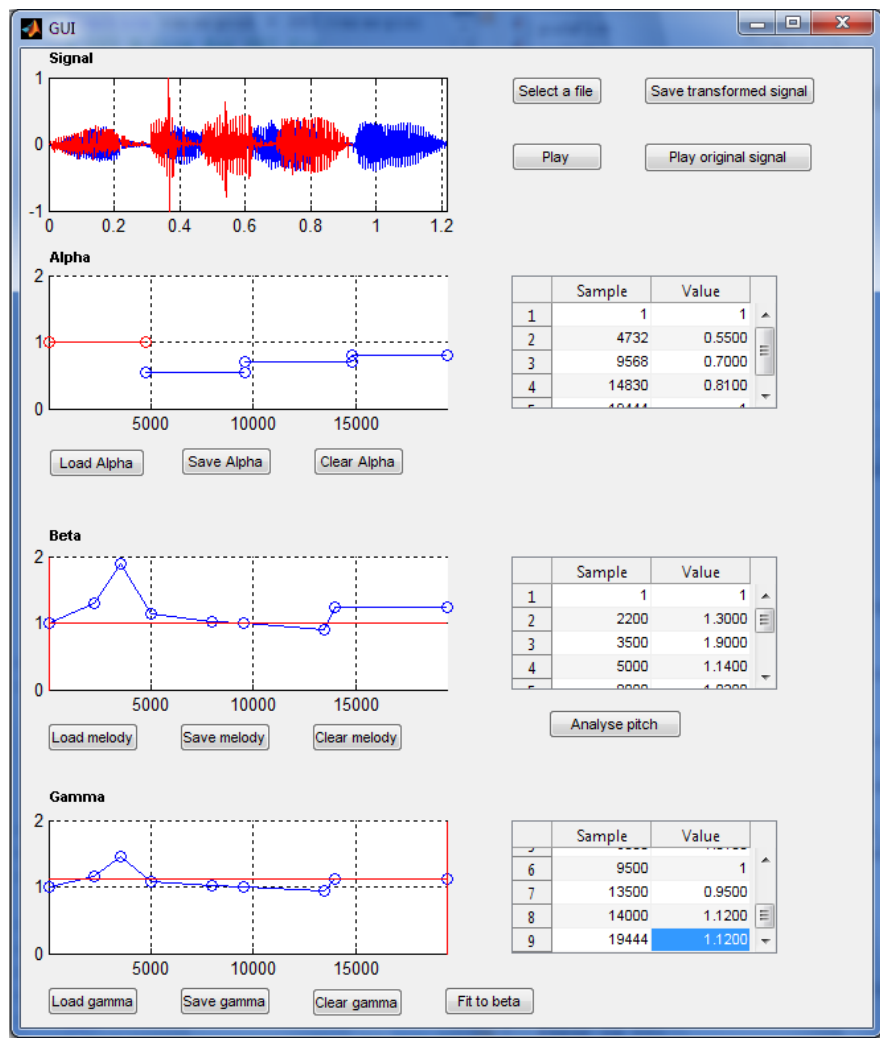

Figure 4. Matlab interface. Transformation for neutral to interrogative tone (red : source signal, blue : transformed signal)

Figure 5 and Table I show that:

- The syllables of the source signal were shortened, and have approximately the same durations as those of the target signal.

- The pitch of the transformed signal is close to that of the target signal.
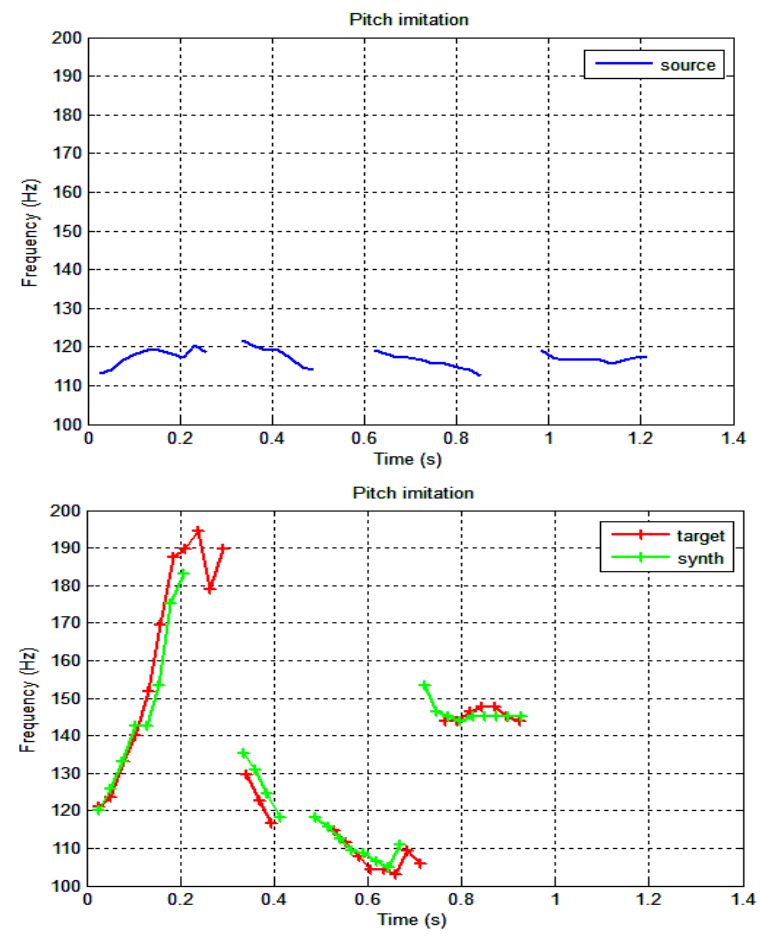

Figure 5. Melodic curves obtained (blue : source signal, red : target, green : transformed signal) 

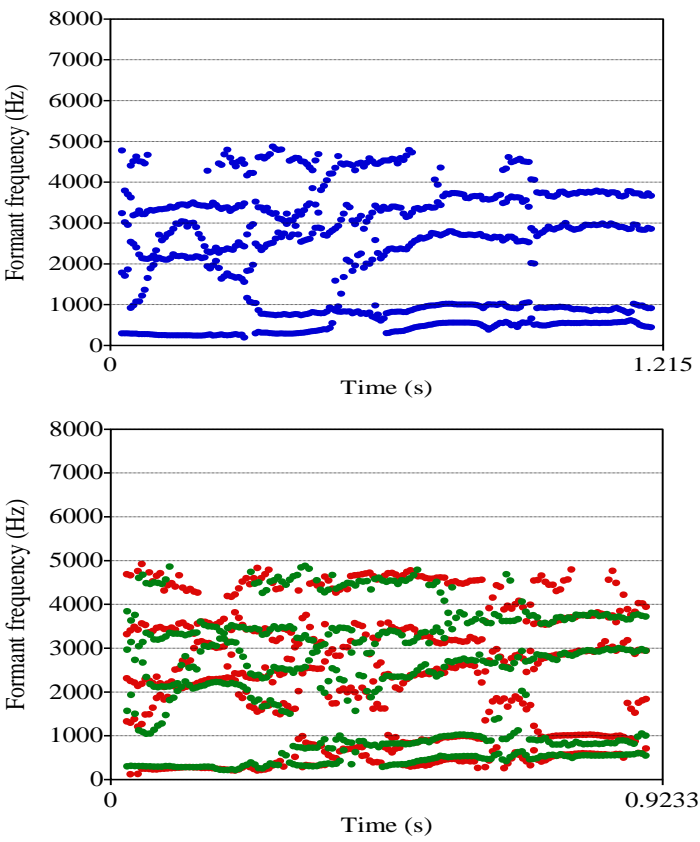

Figure 6. Formantic trajectories (blue: source signal, red: target signal, green: transformed signal)

The pitch was calculated via the autocorrelation method:

$$
\hat{R}_{x y}(m)=\left\{\begin{array}{cc}
\sum_{n=0}^{N-m-1} x_{n+m} y_{n}^{*} & m \geq 0 \\
\hat{R}_{y x}(-m) & m<0
\end{array}\right.
$$

with $x=y$. This method is based on the fact that the autocorrelation of a periodic signal is itself periodic, with the same period. With this knowledge, it is possible to determine the pitch of a vocal signal by looking for the maximum of the autocorrelation function of the vocal signal with itself.

Figure 6 and table II show that the formants of the transformed signal are also close to those of the target signal.

The formants are obtained using PRAAT ${ }^{\circledR}$. The algorithm for computing the formants is to resample the sound to a sampling frequency of twice the value of Maximum formant (which is an adjustable setting). Pre-emphasis is then applied, and then for each analysis window, Praat applies a Gaussian-like window, and computes the LPC coefficients with the algorithm by Burg, as given by [13] and [14] Press et al. (1992).

These results are similar to the ones reported in [11], in which the PSOLA algorithm is used to convert a female voice into a male voice. In this study however, the modification factors of the PSOLA parameters are not constant in time.
Table 1. Fundamental frequencies for the 3 Signals

\begin{tabular}{|c|c|c|c|c|c|}
\hline \multicolumn{2}{|c|}{ Target } & \multicolumn{2}{|c|}{ Source } & \multicolumn{2}{|c|}{ Synth } \\
\hline $\mathrm{t}(\mathrm{s})$ & $\mathrm{fO}(\mathrm{Hz})$ & $\mathrm{t}(\mathrm{s})$ & $\mathrm{fO}(\mathrm{Hz})$ & $\mathrm{t}(\mathrm{s})$ & $\mathrm{fO}(\mathrm{Hz})$ \\
\hline 0.0264 & 120 & 0,0258 & 113 & 0,0262 & 118 \\
\hline 0.0528 & 123 & 0,0517 & 114 & 0,0524 & 126 \\
\hline 0.0791 & 132 & 0,0775 & 116 & 0,0786 & 134 \\
\hline 0.1055 & 139 & 0,1034 & 118 & 0,1048 & 141 \\
\hline 0.1319 & 151 & 0,1292 & 119 & 0,1310 & 145 \\
\hline 0.1583 & 169 & 0,1551 & 119 & 0,1573 & 151 \\
\hline 0.1847 & 187 & 0,1809 & 118 & 0,1835 & 177 \\
\hline 0.2110 & 189 & 0,2068 & 117 & 0,2097 & 187 \\
\hline 0.2374 & 194 & 0,2327 & 120 & 0,2359 & $\mathrm{NaN}$ \\
\hline 0.2638 & 179 & 0,2585 & 118 & 0,2621 & $\mathrm{NaN}$ \\
\hline 0.2902 & 189 & 0,2844 & $\mathrm{NaN}$ & 0,2883 & $\mathrm{NaN}$ \\
\hline 0.3166 & $\mathrm{NaN}$ & 0,3102 & $\mathrm{NaN}$ & 0,3146 & $\mathrm{NaN}$ \\
\hline 0.3429 & 129 & 0,3361 & 121 & 0,3408 & 132 \\
\hline 0.3693 & 122 & 0,3619 & 120 & 0,3670 & 127 \\
\hline 0.3957 & 116 & 0,3878 & 119 & 0,3932 & 122 \\
\hline 0.4221 & $\mathrm{NaN}$ & 0,4137 & 119 & 0,4194 & 120 \\
\hline 0.4485 & $\mathrm{NaN}$ & 0,4395 & 117 & 0,445 & $\mathrm{NaN}$ \\
\hline 0.4748 & 300 & 0,4654 & 114 & 0,4719 & $\mathrm{NaN}$ \\
\hline 0.5012 & $\mathrm{NaN}$ & 0,4912 & 114 & 0,4981 & $\mathrm{NaN}$ \\
\hline 0.5276 & 114 & 0,5171 & $\mathrm{NaN}$ & 0,5243 & 115 \\
\hline 0.5540 & 111 & 0,5429 & 84 & 0,5505 & 112 \\
\hline 0.5804 & 107 & 0,5688 & $\mathrm{NaN}$ & 0,5767 & 110 \\
\hline 0.6067 & 104 & 0,5946 & $\mathrm{NaN}$ & 0,6029 & 107 \\
\hline 0.6331 & 104 & 0,6205 & 119 & 0,6292 & 105 \\
\hline 0.6595 & 103 & 0,6464 & 118 & 0,6554 & 105 \\
\hline 0.6859 & 109 & 0,6722 & 117 & 0,6816 & $\mathrm{NaN}$ \\
\hline 0.7123 & 105 & 0,6981 & 117 & 0,7078 & $\mathrm{NaN}$ \\
\hline 0.7387 & $\mathrm{NaN}$ & 0,7239 & 116 & 0,7340 & 145 \\
\hline 0.7650 & 143 & 0,7498 & 115 & 0,7602 & 144 \\
\hline 0.7914 & 143 & 0,7756 & 115 & 0,7865 & 143 \\
\hline 0.8178 & 146 & 0,8015 & 114 & 0,8127 & 143 \\
\hline 0.8442 & 147 & 0,8274 & 114 & 0,8389 & 143 \\
\hline 0.8706 & 147 & 0,8532 & 112 & 0,8651 & 143 \\
\hline 0.8969 & 145 & 0,8791 & $\mathrm{NaN}$ & 0,8913 & 145 \\
\hline 0.9233 & 143 & 0,9049 & $\mathrm{NaN}$ & & \\
\hline & & 0,9308 & $\mathrm{NaN}$ & & \\
\hline & & 0,9566 & $\mathrm{NaN}$ & & \\
\hline & & 0,9825 & 119 & & \\
\hline & & 1,008 & 117 & & \\
\hline & & 1,034 & 116 & & \\
\hline & & 1,060 & 116 & & \\
\hline & & 1,085 & 116 & & \\
\hline & & 1,111 & 116 & & \\
\hline & & 1,137 & 115 & & \\
\hline & & 1,163 & 116 & & \\
\hline & & 1,189 & 117 & & \\
\hline & & 1,215 & 117 & & \\
\hline
\end{tabular}

Table 2. Formants of the synthetic signal

\begin{tabular}{|c|c|c|c|c|c|c|c|c|c|c|c|c|c|c|}
\hline \multicolumn{5}{|c|}{ Target } & \multicolumn{5}{|c|}{ Source } & \multicolumn{5}{|c|}{ Synth } \\
\hline time (s) & $\begin{array}{c}\mathrm{F} 1 \\
(\mathrm{~Hz})\end{array}$ & $\begin{array}{c}\mathrm{F} 2 \\
(\mathrm{~Hz})\end{array}$ & $\begin{array}{c}\mathrm{F} 3 \\
(\mathrm{~Hz})\end{array}$ & $\begin{array}{c}\mathrm{F} 4 \\
(\mathrm{~Hz})\end{array}$ & time (s) & $\begin{array}{c}\mathrm{F} 1 \\
(\mathrm{~Hz})\end{array}$ & $\begin{array}{c}\mathrm{F} 2 \\
(\mathrm{~Hz})\end{array}$ & $\begin{array}{c}\text { F3 } \\
(\mathrm{Hz})\end{array}$ & $\begin{array}{c}\mathrm{F} 4 \\
(\mathrm{~Hz})\end{array}$ & time $(\mathrm{s})$ & $\begin{array}{c}\mathrm{F} 1 \\
(\mathrm{~Hz})\end{array}$ & $\begin{array}{c}\mathrm{F} 2 \\
(\mathrm{~Hz})\end{array}$ & $\begin{array}{c}\text { F3 } \\
(\mathrm{Hz})\end{array}$ & $\begin{array}{c}\mathrm{F} 4 \\
(\mathrm{~Hz})\end{array}$ \\
\hline 0,111 & 263 & 2121 & 2625 & 3495 & 0,107 & 254 & 2085 & 2545 & 3332 & 0,108 & 285 & 2090 & 2335 & 3311 \\
\hline 0,161 & 258 & 2271 & 3092 & 3567 & 0,157 & 244 & 2179 & 2968 & 3435 & 0,158 & 266 & 2190 & 3082 & 3456 \\
\hline 0,186 & 236 & 2299 & 3018 & 3553 & 0,182 & 242 & 2164 & 2877 & 3449 & 0,183 & 229 & 2196 & 3108 & 3472 \\
\hline 0,211 & 218 & 2302 & 2426 & 3308 & 0,207 & 245 & 2148 & 2571 & 3428 & 0,208 & 259 & 2150 & 2942 & 3448 \\
\hline 0,261 & 231 & 1760 & 2352 & 3372 & 0,257 & 254 & 1725 & 2342 & 3366 & 0,258 & 361 & 1558 & 2345 & 3409 \\
\hline 0,286 & 284 & 1703 & 2779 & 3285 & 0,282 & 243 & 1421 & 2366 & 3413 & 0,283 & 385 & 1490 & 2486 & 3461 \\
\hline 0,311 & 706 & 2404 & 3134 & 3773 & 0,307 & 298 & 1232 & 2490 & 4264 & 0,308 & 356 & 983 & 2516 & 3397 \\
\hline 0,336 & 267 & 771 & 2607 & 3488 & 0,332 & 324 & 869 & 2463 & 3464 & 0,333 & 343 & 829 & 2335 & 3126 \\
\hline 0,361 & 266 & 705 & 2678 & 3156 & 0,357 & 294 & 754 & 2559 & 3203 & 0,358 & 362 & 957 & 2287 & 3407 \\
\hline 0,386 & 641 & 2016 & 2972 & 4574 & 0,382 & 289 & 754 & 2750 & 3091 & 0,383 & 423 & 951 & 2321 & 3474 \\
\hline 0,486 & 343 & 820 & 2293 & 3457 & 0,482 & 428 & 958 & 2888 & 4331 & 0,483 & 385 & 824 & 2395 & 3230 \\
\hline
\end{tabular}




\begin{tabular}{|c|c|c|c|c|c|c|c|c|c|c|c|c|c|c|}
\hline 0,511 & 314 & 866 & 2403 & 3362 & 0,507 & 834 & 1599 & 3056 & 4428 & 0,508 & 464 & 887 & 2487 & 3349 \\
\hline 0,536 & 357 & 907 & 2471 & 3157 & 0,532 & 833 & 1942 & 3194 & 4442 & 0,533 & 510 & 979 & 2549 & 3413 \\
\hline 0,561 & 418 & 957 & 2541 & 3147 & 0,557 & 760 & 2126 & 3218 & 4468 & 0,558 & 526 & 1014 & 2705 & 3634 \\
\hline 0,586 & 416 & 983 & 2594 & 3174 & 0,582 & 669 & 2205 & 3374 & 4461 & 0,583 & 519 & 1006 & 2732 & 3649 \\
\hline 0,611 & 394 & 980 & 2581 & 3199 & 0,607 & 336 & 816 & 2412 & 3433 & 0,608 & 489 & 975 & 2705 & 3645 \\
\hline 0,636 & 937 & 2272 & 3139 & 4551 & 0,632 & 349 & 825 & 2378 & 3311 & 0,633 & 486 & 986 & 2640 & 3646 \\
\hline 0,661 & 618 & 1821 & 2816 & 4009 & 0,657 & 414 & 858 & 2456 & 3275 & 0,658 & 499 & 920 & 2677 & 3701 \\
\hline 0,686 & 794 & 1920 & 3175 & 3778 & 0,682 & 470 & 927 & 2618 & 3365 & 0,683 & 509 & 898 & 2788 & 3651 \\
\hline 0,711 & 482 & 986 & 2759 & 3571 & 0,707 & 510 & 978 & 2714 & 3421 & 0,708 & 538 & 869 & 2813 & 3616 \\
\hline 0,736 & 523 & 995 & 2836 & 3606 & 0,732 & 547 & 1008 & 2750 & 3650 & 0,733 & 541 & 815 & 2832 & 3678 \\
\hline 0,761 & 563 & 1008 & 2873 & 3674 & 0,757 & 556 & 1010 & 2750 & 3717 & 0,758 & 543 & 818 & 2885 & 3743 \\
\hline 0,786 & 583 & 1016 & 2910 & 3731 & 0,782 & 555 & 1001 & 2720 & 3676 & 0,783 & 549 & 816 & 2940 & 3759 \\
\hline \multirow[t]{13}{*}{0,811} & 583 & 1016 & 2936 & 3767 & 0,807 & 538 & 986 & 2714 & 3647 & & & & & \\
\hline & & & & & 0,832 & 494 & 969 & 2700 & 3625 & & & & & \\
\hline & & & & & 0,857 & 487 & 991 & 2643 & 3616 & & & & & \\
\hline & & & & & 0,882 & 534 & 958 & 2622 & 3610 & & & & & \\
\hline & & & & & 0,907 & 578 & 949 & 2558 & 3658 & & & & & \\
\hline & & & & & 0,932 & 503 & 949 & 2650 & 3715 & & & & & \\
\hline & & & & & 0,957 & 502 & 925 & 2792 & 3695 & & & & & \\
\hline & & & & & 0,982 & 532 & 928 & 2884 & 3703 & & & & & \\
\hline & & & & & 1,007 & 547 & 916 & 2896 & 3728 & & & & & \\
\hline & & & & & 1,032 & 548 & 886 & 2885 & 3740 & & & & & \\
\hline & & & & & 1,057 & 551 & 865 & 2945 & 3761 & & & & & \\
\hline & & & & & 1,082 & 551 & 865 & 2961 & 3760 & & & & & \\
\hline & & & & & 1,107 & 559 & 881 & 2948 & 3720 & & & & & \\
\hline
\end{tabular}

\section{Conclusion}

The results obtained with the PSOLA algorithm and the interface are pretty acceptable. The interface has been used to produce various types of sentences. The original sentences were recorded with constant pitch and speech speed, and the modifications of pitch, voice quality and temporal organization were then applied to the signal using the interface.

The voice quality, however, is the most difficult feature to modify using this method. A mere resampling of the waveforms only allows a basic modification of the spectral envelope. It is not possible to alter one formant independently of the others.

Future works are focused on optimizing the interface so that it will be able to automatically imitate the prosodic features of a target signal.

The advantage of this interface lies in its simplicity. It can be used in various domains such as music, cinema, emotion synthesis, correcting speech pathologies, etc.

\section{References}

[1] M. Schübiger: English intonation. Max Niemeyer Verlag, Tübingen (1958)

[2] D. Crystal: Prosodic systems and intonation in English. Cambridge University Press, London (1969)

[3] G. Peeters: Modèles et modification du signal sonore adaptés à ses caractéristiques locales, Thesis, Paris (2001)

[4] [4]. H. Valbret, E. Mouline, J. Tubach : Voice transformation using PSOLA technique, Speech Communication 11 (1992), p. 175-187.
[5] M. Kondoz: Digital Speech, Coding for low bit rate communication systems, Wiley, 2004

[6] M. Jelinek, J. P. Adoul, Frequency-Domain Spectral Envelope Estimation for Low Rate Coding of Speech, ICASSP, 1999.

[7] P. Veprek, M. S. Scordilis, Analysis, enhancement, and evaluation of five pitch determination techniques, Speech Communication 37, 2002

[8] F. Charpentier, Traitement de la parole par Analyse / Synthèse de Fourier application à la synthèse par diphones, Thèse, ENST, Paris, 1988

[9] N. Henrich, B. Doval, C. d'Alessandro, and M.Castellengo. Open quotient measurements on EGG, speech and singing signals. In Proc. 4th International Workshop on Advances in Quantitative Laryngoscopy, Voice and Speech Research, Jena, Apr. 2000

[10] G. Peeters. Analyse et synthèse des sons musicaux par la méthode PSOLA. In JIM98- Workshop, Agelonde, France, Mai 1998.

[11] A. Mousa. Voice Conversion Using Pitch Shifting Algorithm by Time Stretching with PSOLA and Re-Sampling. Journal of Electrical Engineering, Vol 61, NO. 1, 2010, p. 57-61

[12] P. Dutilleux, G. De Poli, U. Zölzer, DAFX - Digital Audio Effects, U. Zölzer, Ed. John Wiley \& Sons, Sussex, England, 2002, p. 201-234.

[13] D. Childers, Modern Spectrum Analysis, IEEE Press, Piscateway, New Jersey, U.S., 1978, p. 252-255.

[14] W.H. Press, S.A. Teukolsky, W.T. Vetterling, B.P. Flannery, Numerical Recipes in $C$ : the art of scientific computing, Second Edition, Cambridge University Press, 1992. 